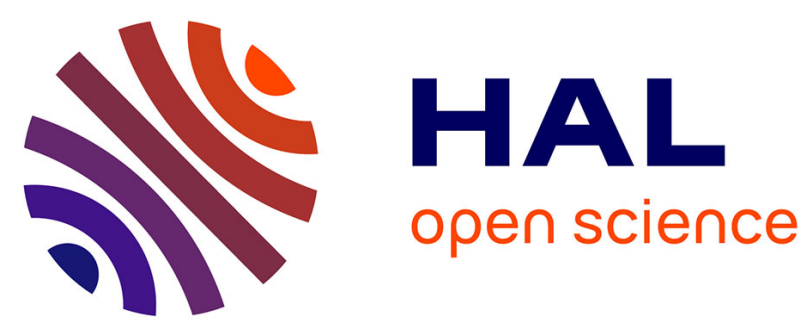

\title{
Computing Period and Shape of Oscillations in Piecewise Linear Lur'e Systems: a Complementarity Approach
}

Valentina Sessa, Luigi Iannelli, Vincent Acary, Bernard Brogliato, Francesco Vasca

\section{To cite this version:}

Valentina Sessa, Luigi Iannelli, Vincent Acary, Bernard Brogliato, Francesco Vasca. Computing Period and Shape of Oscillations in Piecewise Linear Lur'e Systems: a Complementarity Approach. CDC 2013 - 52nd IEEE Conference on Decision and Control, Dec 2013, Firenze, Italy. pp.4680-4685, 10.1109/CDC.2013.6760622 . hal-00870282

\section{HAL Id: hal-00870282 \\ https://hal.inria.fr/hal-00870282}

Submitted on 6 Oct 2013

HAL is a multi-disciplinary open access archive for the deposit and dissemination of scientific research documents, whether they are published or not. The documents may come from teaching and research institutions in France or abroad, or from public or private research centers.
L'archive ouverte pluridisciplinaire HAL, est destinée au dépôt et à la diffusion de documents scientifiques de niveau recherche, publiés ou non, émanant des établissements d'enseignement et de recherche français ou étrangers, des laboratoires publics ou privés. 


\title{
Computing Period and Shape of Oscillations in Piecewise Linear Lur'e Systems: a Complementarity Approach
}

\author{
Valentina Sessa $^{\diamond}$, Luigi Iannelli ${ }^{\diamond}$, Vincent Acary ${ }^{\natural}$, Bernard Brogliato ${ }^{b}$ and Francesco Vasca ${ }^{\diamond}$
}

\begin{abstract}
Autonomous piecewise linear systems in the Lur'e form may exhibit periodic steady-state oscillations. For many practical systems belonging to this class the period and the shape of the oscillation is difficult to be predicted a priori. In this paper the complementarity approach is used to tackle the issue. The complementarity formalism is used to represent the closed-loop system and a phase condition acting as an anchor equation for the periodic solution. By discretizing the dynamics a mixed complementarity problem is formulated. The corresponding solution provides an accurate prediction of the steady-state oscillation and its period. Numerical results show the effectiveness of the proposed technique for the computation of stable and sliding periodic solutions. The analysis of the steady-state solution of a Colpitts oscillator is considered as an illustration.
\end{abstract}

\section{INTRODUCTION}

A piecewise linear (PWL) system in Lur'e form, as the one shown in Fig. 1, can be represented as the feedback interconnection of a linear time-invariant dynamical system $\Sigma$ with a piecewise linear static relation $\mathcal{R}$ which relates the system output, $y$ and the opposite of the system input, $\lambda$. This class of dynamical systems has attracted a considerable interest in the literature, because they may exhibit several interesting behaviors. For instance, such systems tend to periodically oscillate also without external excitation. This means that the period related to the periodic solution is difficult to be a priori predicted. In the literature various mathematical methods, basically classified as time-domain or frequencydomain have been proposed to compute the steady-state periodic solution and its period. Time-domain approaches are based mainly on the so-called shooting method which determines the initial condition and the period for the periodic solution by solving a sequence of nonlinear initial value problems with the Newton-Raphson method, [1], [2], [3]. The main drawback of this method is the evaluation of the sensitivity matrix, which is often computationally expensive and becomes even more complicated for nonsmooth systems, [4], [5]. In frequency-domain, harmonic balance is the classical technique used for determining the steady-state behaviour of nonlinear autonomous systems that exhibit a single periodic attractor, [6]. The describing function (i.e. harmonic balance with a single harmonic) provides simple results about the existence of a periodic oscillation and its parameters (the amplitude and the period), but it is

\footnotetext{
$\diamond$ Department of Engineering, University of Sannio, Piazza Roma 21, 82100 Benevento, Italy e-mail: valentina.sessa, luigi.iannelli, vasca@unisannio.it

b INRIA, Bipop team-project, Inovallee de Montbonnot, 655 avenue, Europe 38334 Saint Ismier cedex, France e-mail: vincent. acary, bernard.brogliatodinrialpes.fr
}

not able to accurately predict them particularly when the system under consideration does not satisfy the assumption of filtering out the higher-order harmonics, [7]. In [8] a mixed time-frequency-domain approach is used to analyze a nonlinear oscillator by linearizing the system along the solution predicted by the harmonic balance technique and then by computing the Floquet's multipliers by using the time-domain numerical algorithm.

The recent literature has shown that the complementarity framework can be useful for investigating PWL systems, [9], [10]. In [11], [12] and [13], the complementarity framework is used for the computation of periodic solutions in autonomous Lur'e systems. In those papers it has been shown how the linear or mixed linear complementarity representation of the feedback characteristic allows to represent the discretized closed-loop system as a linear complementarity system. Then the solution of the corresponding complementarity problem allows to obtain the periodic solution of the discretized system. The main drawback of the previous approaches is that the period must be known a priori or, at least, a certain estimation of the period must be computed by using, for instance, the describing function technique. Instead in this paper the period is considered as a further unknown and it is computed together with the periodic solution by constructing a suitable mixed complementarity problem (MCP).

The paper is organized as follows. In Section II some preliminaries about the solution concept for autonomous PWL systems and the complementarity theory are presented. In Section III we show how to formulate a MCP in order to compute a periodic solution together with the period. In Section IV three interesting applications are considered: a saturation Lur'e system, a relay feedback system which exhibits a periodic solution with sliding, and a practical electronic oscillator. The numerical results demonstrate the effectiveness of the proposed approach. The paper is concluded in Section V.

\section{PWL LuR'e Systems And Periodic Solutions}

The Lur'e system in Fig. 1, for the analysis considered in this paper, can be represented in the following state space form

$$
\begin{aligned}
& \dot{x}=A x+B(-\lambda)+g \\
& y=C x+D(-\lambda)+h \\
& (y, \lambda) \in \mathcal{R}
\end{aligned}
$$

where $(A, B, C, D)$ is a minimal state space realization with $A \in \mathbb{R}^{n \times n}, B \in \mathbb{R}^{n \times m}, C \in \mathbb{R}^{m \times n}, D \in \mathbb{R}^{m \times m}, g \in$ 


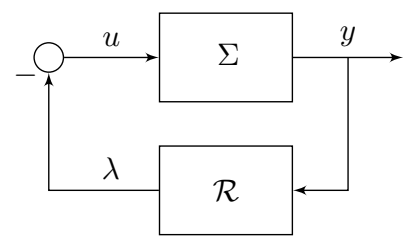

Fig. 1. Block diagram of a Lur'e system.

$\mathbb{R}^{n \times 1}$ and $h \in \mathbb{R}^{m \times 1}$ all being constant, $\mathcal{R}$ is a static PWL relation that includes also set-valued characteristics, and the time derivative is meant almost everywhere.

Definition 1: A solution of the system (1) is any absolutely continuous function $x(t):\left[t_{0},+\infty\right) \mapsto \mathbb{R}^{n}$ that satisfies (1) for almost every $t \geq t_{0}$, given an initial condition $x\left(t_{0}\right)$.

We assume that, for every initial condition $x\left(t_{0}\right)$, (1) has at least one solution.

Definition 2: A solution $x$ of the system (1) is periodic if there exists $T$ such that $x(t+T)=x(t)$ for any $t \in \mathbb{R}$.

The PWL feedback relation $\mathcal{R}$ is represented by using the mixed complementarity problem formulation. A MCP is defined as follows, [14].

Problem 1: Given a function $f: \mathbb{R}^{r} \rightarrow \mathbb{R}^{r}$ and lower and upper bounds $l, u \in \mathbb{R}^{r} \cup\{-\infty,+\infty\}^{r}$,

$$
\begin{array}{cl}
\text { find } & z \in \mathbb{R}^{r}, w \in \mathbb{R}_{+}^{r}, v \in \mathbb{R}_{+}^{r} \\
\text { s.t. } & f(z)=w-v \\
& l \leq z \leq u,(z-l)^{\top} w=0,(u-z)^{\top} v=0 .
\end{array}
$$

When $f(z)$ is affine, Problem 1 defines a mixed linear complementarity problem (MLCP), that can be defined as follows.

Problem 2: Given a vector $q \in \mathbb{R}^{r}$, a real matrix $M \in$ $\mathbb{R}^{r \times r}$ and lower and upper bounds $l, u \in \mathbb{R}^{r} \cup\{-\infty,+\infty\}^{r}$,

$$
\begin{array}{ll}
\text { find } & z \in \mathbb{R}^{r}, w \in \mathbb{R}_{+}^{r}, v \in \mathbb{R}_{+}^{r} \\
\text { s. t. } & M z+q=w-v \\
& l \leq z \leq u,(z-l)^{\top} w=0,(u-z)^{\top} v=0 .
\end{array}
$$

A linear complementarity problem can be formulated as Problem 2 by setting the lower bound equal to zero and the upper bound equal to infinity: in this case $z$ and $w$ are the usual complementarity variables.

Problem 2 can be used to represent the class of relation $(y, \lambda)$ considered in this paper, where $y$ is the input of the relation and $\lambda$ is the output. For instance, by choosing $z=\lambda$ and $q=-y$, Problem 2 can be rewritten in the following form.

Problem 3: Given a vector $y \in \mathbb{R}^{m}$, a real matrix $M \in \mathbb{R}^{m \times m}$ and lower and upper bounds $l_{\lambda}, u_{\lambda} \in \mathbb{R}^{m} \cup$ $\{-\infty,+\infty\}^{m}$,

$$
\begin{array}{ll}
\text { find } & \lambda \in \mathbb{R}^{m}, w_{\lambda} \in \mathbb{R}_{+}^{m}, v_{\lambda} \in \mathbb{R}_{+}^{m} \\
\text { s. t. } & M \lambda-y=w_{\lambda}-v_{\lambda} \\
& l_{\lambda} \leq \lambda \leq u_{\lambda},\left(\lambda-l_{\lambda}\right)^{\top} w_{\lambda}=0,\left(u_{\lambda}-\lambda\right)^{\top} v_{\lambda}=0 .
\end{array}
$$

A wide class of PWL characteristics can be represented in the mixed linear complementarity framework, [13], such as the unitary single-input single-output saturation characteristic that can be represented by using Problem 3 with $M=1$, $l_{\lambda}=-1$ and $u_{\lambda}=1$. See [15] for a more exhaustive analysis about PWL representations in the complementarity framework.

Let $x(t)$ be a nonconstant periodic solution of (1) with the unknown period $T$. Since the phase of a periodic solution belonging to an autonomous systems is not fixed, any time translation of the periodic solution gives another 'different' periodic solution. In other words, if the closed-loop system admits a periodic solution, it admits an infinite number of periodic solutions each one differing from the others by a translation in time. In order to fix the initial phase of the periodic solution, one more equation is required. In the literature it has been proposed to add an anchor equation that allows to block the profile of the periodic solution. Such a normalization is frequently called phase condition. The most known anchor equation is the orthogonality condition that is successfully used in the continuation method for the bifurcation analysis, [16] and it is also applied to the shooting method, [17]. For our approach it seems to be more natural the phase condition proposed in [18]:

$$
\dot{x}_{j}(\bar{t})=c_{j}^{\top}(A x(\bar{t})-B \lambda(\bar{t})+g)=0,
$$

where $x_{j}$ is a generic $j$-th component of the state at a chosen time instant $\bar{t} \in[0, T]$ and $c_{j}^{\top} \in \mathbb{R}^{1 \times n}$ is a zero vector whose $j$-th element is 1 . Note that the index $j$ can be chosen arbitrarily in the case of sufficiently smooth solutions. Indeed, in the case of periodic solutions $x(t) \in C^{1}$, the time derivative of each state variable must be zero at least at one time instant $\bar{t} \in[0, T]$. If $x(t) \in C^{0}$ as in the case of the sliding motion, we have to pay attention to the fact that the time derivative can jump. We should assume that there exists a small time interval in which $\dot{x}_{j}=0$. This paper will not take into account solutions with jumps in the state.

The dynamic model and the anchor equation can be normalized with respect to the unknown period by using the time scaling $t=T \tau$, where $\tau$ is a dimensionless time variable. Then the system (1) can be written as

$$
\begin{aligned}
& x^{\prime}=T A x+T B(-\lambda)+T g \\
& y=C x+D(-\lambda)+h \\
& (y, \lambda) \in \mathcal{R}
\end{aligned}
$$

where $x^{\prime}$ is the derivative with respect to $\tau$. A periodic solution of (1) with period $T$ will correspond to a periodic solution of (6) with period equal to 1 . For the system (6) the phase condition can be written as

$$
x_{j}{ }^{\prime}(\bar{\tau})=T c_{j}^{\top}(A x(\bar{\tau})-B \lambda(\bar{\tau})+g)=0
$$

for some $\bar{\tau} \in[0,1]$.

\section{MCP FOR THE COMPUTATION OF A PERIODIC SOLUTION AND ITS PERIOD}

The MCP formulation for the computation of periodic solutions in Lur'e systems requires some preliminary no- 
tations. In the following we assume that $\operatorname{col}(\cdot)$ indicates a vector obtained by stacking in a unique column the column vectors in its argument, $I_{N}$ denotes the $N \times N$ identity matrix and $1_{N}, \infty_{N}$ are the $N$-dimensional vectors of ones and of infinity, respectively. The symbol $\otimes$ indicates the Kronecker product.

By discretizing (6) with the $(\theta, \gamma)$-technique, [19] and a sampling period $1 / N, N$ being an integer, we get the following discrete-time system:

$$
\begin{aligned}
& \left(I_{n}-\frac{T}{N} \theta A\right) x_{k}-\left(I_{n}+\frac{T}{N}(1-\theta) A\right) x_{k-1}= \\
& \quad-\gamma \frac{T}{N} B \lambda_{k}-\frac{T}{N}(1-\gamma) B \lambda_{k-1}+\frac{T}{N} g \\
& y_{k}=C x_{k}-D \lambda_{k}+h \\
& \left(y_{k}, \lambda_{k}\right) \in \mathcal{R}
\end{aligned}
$$

with $k=1, \ldots, N, \theta \in[0,1]$ and $\gamma \in[0,1]$. By opportunely rearranging (8b) for all samples $k=1, \ldots, N$, by using the periodicity constraint $x_{0}=x_{N}$ and $\lambda_{0}=\lambda_{N}$ and by adding the discretized version of the phase constraint (7), we obtain a MCP which can be represented in the form of Problem 1 with

$$
\begin{aligned}
z & =\operatorname{col}(\bar{\lambda}, \bar{x}, T) \\
f(z) & =\operatorname{col}\left(f_{\lambda}(z), f_{x}(z), f_{T}(z)\right) \\
l & =\operatorname{col}\left(l_{\bar{\lambda}}, l_{\bar{x}}, l_{T}\right)=\operatorname{col}\left(l_{\lambda} \cdot 1_{N},-\infty_{N} \cdot n,-\infty\right) \\
u & =\operatorname{col}\left(u_{\bar{\lambda}}, u_{\bar{x}}, u_{T}\right)=\operatorname{col}\left(u_{\lambda} \cdot 1_{N},+\infty_{N} \cdot n,+\infty\right)
\end{aligned}
$$

with $\bar{x}=\operatorname{col}\left(x_{1}, x_{2}, \ldots, x_{N}\right), \bar{\lambda}=\operatorname{col}\left(\lambda_{1}, \lambda_{2}, \ldots, \lambda_{N}\right)$ and $\bar{y}=\operatorname{col}\left(y_{1}, y_{2}, \ldots, y_{N}\right)$ and where

$$
\begin{aligned}
f_{\lambda}(z) & =-\bar{C} \bar{x}+(\bar{D}+\bar{M}) \bar{\lambda}-\bar{h} \\
f_{x}(z) & =\left(I_{n}-\frac{T}{N} \theta A\right) x_{k}-\left(I_{n}+\frac{T}{N}(1-\theta) A\right) x_{k-1}+ \\
& +\gamma \frac{T}{N} B \lambda_{k}+\frac{T}{N}(1-\gamma) B \lambda_{k-1}-\frac{T}{N} g, k=1, \ldots, N
\end{aligned}
$$

$f_{T}(z)=c_{j}^{\top}\left(x_{\hat{k}}-x_{\hat{k}-1}\right)$ for a chosen $\hat{k}$ and a chosen $j$

with

$$
\begin{array}{llrl}
\bar{M} & =I_{N} \otimes M, & & \bar{C}=I_{N} \otimes C \\
\bar{D} & =I_{N} \otimes D, & & \bar{h}=1_{N} \otimes h .
\end{array}
$$

Note that the choice of the unbounded limits on the variables $\bar{x}$ and $T$ implies that $f_{x}(z)$ and $f_{T}(z)$ are equal to zero.

Depending on the relation $(y, \lambda)$, constant solutions of (1) are also possible. For instance, when $g$ and $h$ are zero and $(0,0) \in(y, \lambda)$, the origin is also a solution (an equilibrium) of the Lur'e system. We are interested in the solution that corresponds to a nonconstant one, i.e. a solution that corresponds to a periodic solution. Since the phase constraint (7) does not exclude the trivial solution (and more in general constant solutions), one must add a further constraint which excludes the zero solution. To this aim the following condition can be used

$$
x_{(\bullet, \hat{i})}^{\top} x_{(\bullet, \hat{i})}>0
$$

for some chosen $\hat{i}$, where $x_{(\bullet, \hat{i})} \in \mathbb{R}^{N \times 1}$ is the vector obtained by collecting all samples of the $\hat{i}$-th component of the state space. It is necessary to represent (12) in a suitable complementarity form. A possible complementarity representation of (12) is the following:

$$
\begin{array}{ll}
\text { find } & \rho \in \mathbb{R}, w_{\rho} \in \mathbb{R}_{+}, v_{\rho} \in \mathbb{R}_{+} \\
\text {s. t. } & x_{(\bullet, \hat{i})}^{\top} x_{(\bullet, \hat{i})}-\epsilon=w_{\rho}-v_{\rho} \\
& l_{\rho} \leq \rho \leq u_{\rho},\left(\rho-l_{\rho}\right) w_{\rho}=0,\left(u_{\rho}-\rho\right) v_{\rho}=0,
\end{array}
$$

where $\epsilon$ is a small positive parameter, $l_{\rho}=0$ and $u_{\rho}=+\infty$ that implies $v_{\rho}=0$. Indeed the nonnegativity of the variable $w_{\rho}$ in (13b) implies that $x_{(\bullet, \hat{i})}^{\top} x_{(\bullet, \hat{i})}$ must be different from zero.

The approach presented above for the elimination of the trivial solution can be simply extended for the elimination of a nonzero constant solution.

Now, we are able to formulate the MCP that allows to compute the nontrivial periodic solution and its period.

Problem 4: Given the matrices $A, B, C, D, g, h$ of the model (1), the matrix $M$ of the feedback relation representation (4), the discretization technique parameters $(\theta, \gamma)$ and the number of discrete samples $N$

$$
\begin{aligned}
& \text { find } \bar{\lambda} \in \mathbb{R}^{N \cdot m}, \bar{x} \in R^{N \cdot n}, T, \rho \in \mathbb{R} \\
& w_{\lambda}, v_{\lambda} \in \mathbb{R}_{+}^{N \cdot m}, w_{x}, v_{x} \in \mathbb{R}_{+}^{N \cdot n}, \\
& w_{T}, v_{T}, w_{\rho}, v_{\rho} \in \mathbb{R}_{+} \\
& \text {s. t. }-\bar{C} \bar{x}+(\bar{D}+\bar{M}) \bar{\lambda}-\bar{h}=w_{\lambda}-v_{\lambda} \\
& \left(I_{n}-\frac{T}{N} \theta A\right) x_{k}-\left(I_{n}+\frac{T}{N}(1-\theta) A\right) x_{k-1}+ \\
& +\gamma \frac{T}{N} B \lambda_{k}+\frac{T}{N}(1-\gamma) B \lambda_{k-1}-\frac{T}{N} g=w_{x}-v_{x}, \\
& k=1, \ldots, N \\
& c_{j}^{\top}\left(x_{\hat{k}}-x_{\hat{k}-1}\right)=w_{T}-v_{T} \text { for a chosen } \hat{k} \\
& \text { and a chosen } j \\
& x_{(\bullet, \hat{i})}^{\top} x_{(\bullet, \hat{i})}-\epsilon=w_{\rho}-v_{\rho} \text { for a chosen } \hat{i} \\
& l_{\bar{\lambda}} \leq \bar{\lambda} \leq u_{\bar{\lambda}},\left(\bar{\lambda}-l_{\bar{\lambda}}\right)^{\top} w_{\lambda}=0,\left(u_{\bar{\lambda}}-\bar{\lambda}\right)^{\top} v_{\lambda}=0 \\
& l_{\bar{x}} \leq \bar{x} \leq u_{\bar{x}},\left(\bar{x}-l_{\bar{x}}\right)^{\top} w_{x}=0,\left(u_{\bar{x}}-\bar{x}\right)^{\top} v_{x}=0 \\
& l_{T} \leq T \leq u_{T},\left(T-l_{T}\right) w_{T}=0,\left(u_{T}-T\right) v_{T}=0 \\
& l_{\rho} \leq \rho \leq u_{\rho},\left(\rho-l_{\rho}\right) w_{\rho}=0,\left(u_{\rho}-\rho\right) v_{\rho}=0
\end{aligned}
$$

with $x_{0}=x_{N}, \lambda_{0}=\lambda_{N}, \bar{C}, \bar{D}, \bar{M}, \bar{h}$ given by (11), lower bounds $l_{\bar{\lambda}}, l_{\bar{x}}, l_{T}$ given by (9c), $l_{\rho}=0$ and upper bounds $u_{\bar{\lambda}}, u_{\bar{x}}, u_{T}$ given by (9d), $u_{\rho}=+\infty$. 


\section{NumeriCAL RESUlts}

The effectiveness of the proposed technique for the computation of period and waveform of oscillations is shown by considering different examples: a saturation feedback system, a relay feedback system with sliding motion and the Colpitts electronic oscillator. The solution of Problem 4 for a given $N$ is compared with that obtained with a large value of $\mathrm{N}$, say $N_{\max }$, which is chosen for a reasonable computational effort, e.g. time elapsed less than 1 hour. According to the numerical simulation schemes presented in [19], we show numerical results obtained by varying the values of $\theta$ and $\gamma$. Problem 4 is solved by using the efficient PATH solver [14], that is a generalization of the Newton method for nonsmooth problems, so it requires an initial condition, say $\left[\bar{\lambda}^{0}, \bar{x}^{0}, T^{0}, \rho^{0}\right]$. The default starting point in the PATH solver is a zero vector. In order to help the solver in avoiding the trivial solution, we choose as initial condition a vector different from the trivial one and, in particular, we decided to choose as a guess value $T^{0}$, the one computed by means of the describing function.

\section{A. A limit cycle in a saturation feedback system}

Consider the system (1) with the following matrices

$$
\begin{array}{lll}
A=\left[\begin{array}{ccc}
0 & 1 & 0 \\
0 & 0 & 1 \\
0 & -3 & -4
\end{array}\right], & B=\left[\begin{array}{c}
0 \\
0 \\
20
\end{array}\right], & g=\left[\begin{array}{l}
0 \\
0 \\
0
\end{array}\right] \\
C=\left[\begin{array}{lll}
1 & 0 & 0
\end{array}\right], & D=0, & h=0
\end{array}
$$

and the piecewise linear feedback $(y, \lambda)$ being a unitary saturation. Since the relation $\mathcal{R}$ is a Lipschitz continuous function of $x$, the solution is expected to be of class $C^{1}$. See [13] for the definition and a graphical representation of the limit cycle. Figure 2 shows the evolution of the error obtained using different discretization schemes. In particular, in Fig. 2(a) the error is defined as the difference between $T_{\text {ref }}$, that is the value of the period computed by solving Problem 4 with $N=N_{\max }=8500$, and the value of period obtained with the same discretization scheme, but by varying $N$ from 200 to 1000 samples. The same reasoning is carried out to obtain the results in Fig. 2(b) where the maximum value of the component $x_{1}$ is considered. For $\theta=1, \gamma=1$, we obtain as expected an order 1 convergence which is represented by straight lines on the graph. For values of $\theta=0.5$ and $\gamma$ towards 0.5 the accuracy is improved but we do not achieve order 2 when $\gamma=0.5$. This may be mainly due to the lack of regularity of the computed solution. This phenomenon is generally observed when higher order method is used for a solution with limited smoothness (see [20, §9.1]).

\section{B. A limit cycle with sliding motion}

Consider a relay feedback system, in which the dynamical system is represented by the following matrices

$$
\begin{aligned}
& A=\left[\begin{array}{lll}
-3 & 1 & 0 \\
-3 & 0 & 1 \\
-1 & 0 & 0
\end{array}\right], \quad B=\left[\begin{array}{c}
1 \\
-2 \\
1
\end{array}\right], \quad g=\left[\begin{array}{l}
0 \\
0 \\
0
\end{array}\right] \\
& C=\left[\begin{array}{lll}
1 & 0 & 0
\end{array}\right], \quad D=0, \quad h=0 \text {. }
\end{aligned}
$$

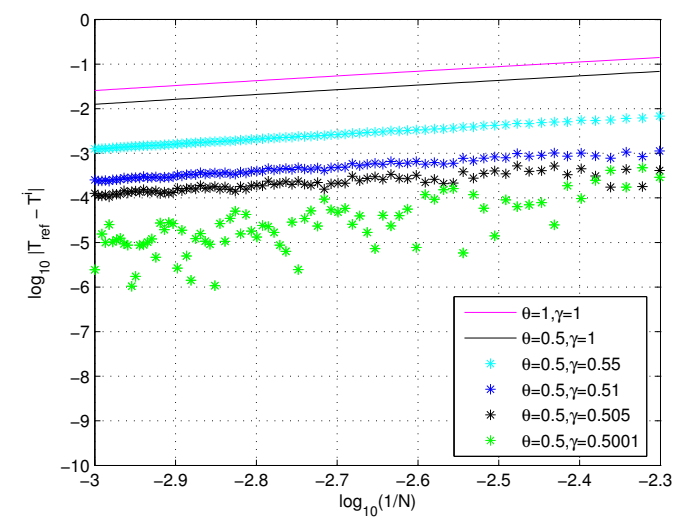

(a)

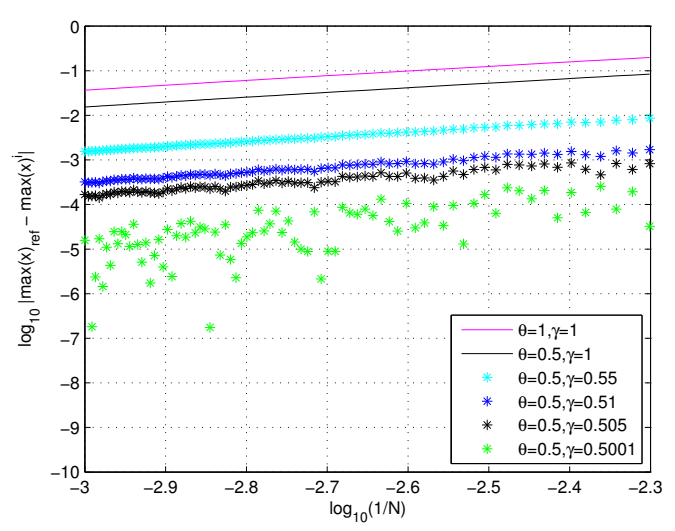

(b)

Fig. 2. Lur'e system with feedback saturation function. Error in the steady-state solution computation obtained with different time discretization schemes and with $N \in[200,1000]:$ (a) error in the period (b) error in the trajectory.

By using Problem 3 with the parameters $M=0, l_{\lambda}=-1$ and $u_{\lambda}=1$ the relay characteristic can be easily represented. In [21], it is shown that the state evolution of this system partially evolves along the switching surface $\left\{x \in \mathbb{R}^{n}\right.$ : $C x=0\}$. This type of solution is known as sliding solution. In this case the solution $x(t)$ is absolutely continuous, so since the smoothness of the solution is one degree lower with respect to the previous example, the time-stepping scheme is chosen by fixing $\gamma=1$ and by varying only $\theta$. Qualitatively the same comments about the accuracy of the error can be repeated, see Fig. 3.

\section{Colpitts oscillator}

Consider the Colpitts oscillator, which is shown in Fig. 4, where the bipolar junction transistor is represented by using the Ebers-Moll model. It is known in the literature that with appropiate values of parameters, this circuit presents a stable periodic oscillation, [22]. The circuit model can be simply written by applying the Kirchhoff currents and voltages laws to the equivalent circuit in Fig. 4: 


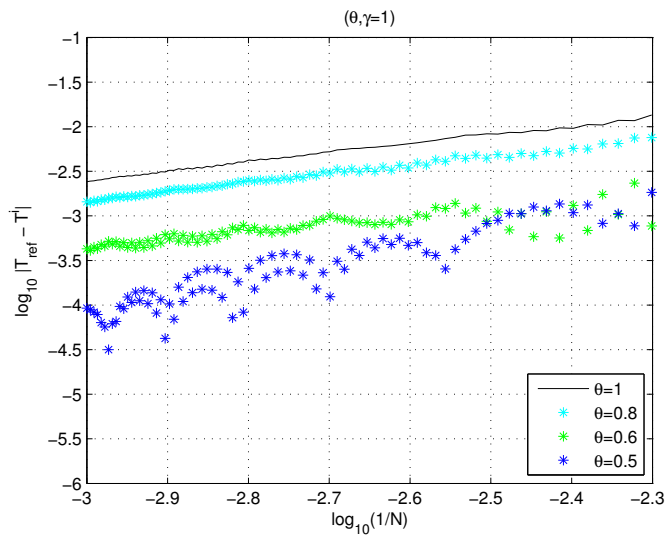

(a)

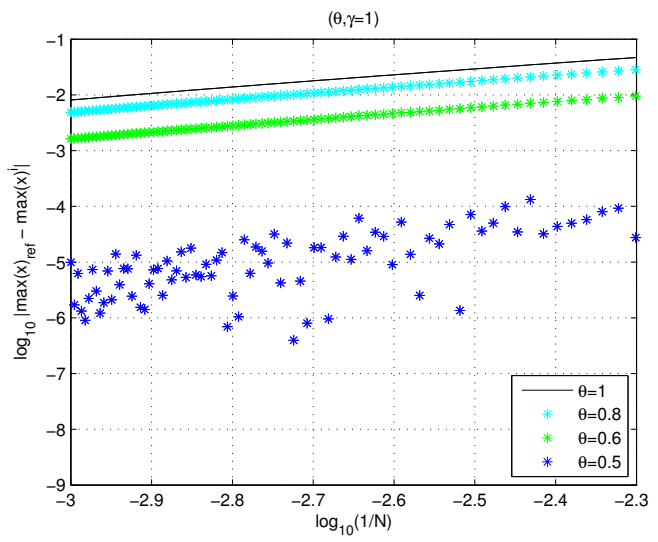

(b)

Fig. 3. Relay feedback system. Error in the steady-state solution computation obtained with different time discretization schemes and with $N \in$ [200, 1000]: (a) error in the period (b) error in the trajectory.

$$
\begin{aligned}
C_{1} \dot{x}_{1}= & -\frac{1}{R_{C}} x_{1}-\frac{1}{R_{C}} x_{2}+x_{3}+\frac{1}{R_{C}} V_{C C}-i_{C} \\
C_{2} \dot{x}_{2}= & -\frac{1}{R_{C}} x_{1}-\left(\frac{1}{R_{C}}+\frac{1}{R_{B}}+\frac{1}{R_{E}}\right) x_{2}+x_{3} \\
& +\frac{1}{R_{B}} \lambda_{2}+\frac{1}{R_{C}} V_{C C}-\frac{1}{R_{E}} V_{E E} \\
L \dot{x}_{3}= & -x_{1}-x_{2}+V_{C C},
\end{aligned}
$$

and the transistor collector and base currents are given respectively by

$$
\begin{aligned}
i_{C} & =-\frac{1-\alpha_{F} \alpha_{R}}{1-\alpha_{F}} \lambda_{1}+\frac{\alpha_{F}}{1-\alpha_{F}} i_{B} \\
i_{B} & =\frac{1}{R_{B}}\left(\lambda_{2}-x_{2}\right) .
\end{aligned}
$$

By substituting (18) in (17) and by choosing as outputs

$$
\begin{aligned}
& y_{1}=-x_{1} \\
& y_{2}=\frac{1}{\left(1-\alpha_{F}\right) R_{B}} x_{2}
\end{aligned}
$$

one can write the dynamic system in the form of (1a)-(1b). The feedback relation $(y, \lambda)$ can be obtained by applying the Kirchhoff laws to the equivalent circuit and by using the

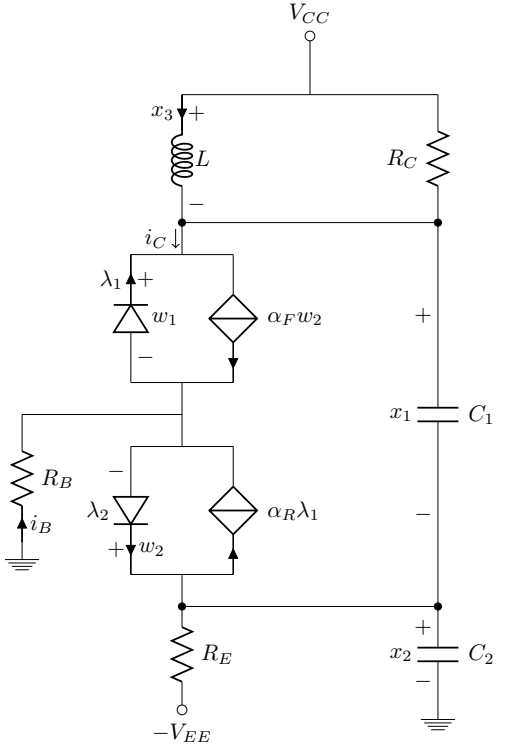

Fig. 4. Colpitts oscillator: $R$ are resistances; $C$ are capacitors; $L$ is the inductance; $V_{E E}$ and $V_{C C}$ are constant voltage sources; $\alpha_{R}$ and $\alpha_{F}$ are transistor parameters; the subscripts $B, E$ and $C$ are used for the transistor base, emitter and collector nodes, respectively.

ideal diode complementarity models

$$
\lambda_{i} w_{i}=0, \lambda_{i} \in \mathbb{R}_{+}, w_{i} \in \mathbb{R}_{+}
$$

with $i=1,2$. Indeed, the choice of the output as in (19), allows to rewrite the relation $(y, \lambda)$ in the form of Problem 3 with

$$
\begin{aligned}
M & =\left[\begin{array}{cc}
0 & 1 \\
-\frac{1-\alpha_{R}}{1-\alpha_{F}} & \frac{1}{\left(1-\alpha_{F}\right) R_{B}}
\end{array}\right] \\
l_{\lambda} & =\operatorname{col}(0,0), u_{\lambda}=\operatorname{col}(+\infty,+\infty) .
\end{aligned}
$$

Consider the following parameters $R_{C}=10 \Omega, L=0.1 \mathrm{H}$, $C_{1}=2 \mathrm{~F}, C_{2}=0.8 \mathrm{~F}, R_{E}=20 \Omega, R_{B}=0.5 \Omega, \alpha_{F}=$ $0.99, \alpha_{R}=0.015, V_{C C}=10 \mathrm{~V}$ and $V_{E E}=20 \mathrm{~V}$. The collector and emitter voltages computed with the pair $(\theta=$ $0.5, \gamma=0.5)$ and $N=N_{\max }=900$ are shown in Fig. 5(a), while Fig. 5(b) shows the steady-state values of the collector and base currents. Figure 6 shows that the error decreases when the number of the samples is increased confirming the effectiveness of the proposed approach.

\section{CONCLUSION}

In this paper, a suitable mixed complementarity problem is formulated in order to compute periodic solutions in autonomous Lur'e systems. A phase condition is used and a nonlinear version of the complementarity problem is formulated in order to compute also the period of the periodic solution. A method that allows to eliminate the constant solutions has been also presented. Numerical examples are considered as case studies: a saturation feedback system, a relay feedback system with sliding motion and an electronic oscillator. The simulations, implemented by using different discretization schemes, show that the error between the real solution and the one computed by varying the number of 


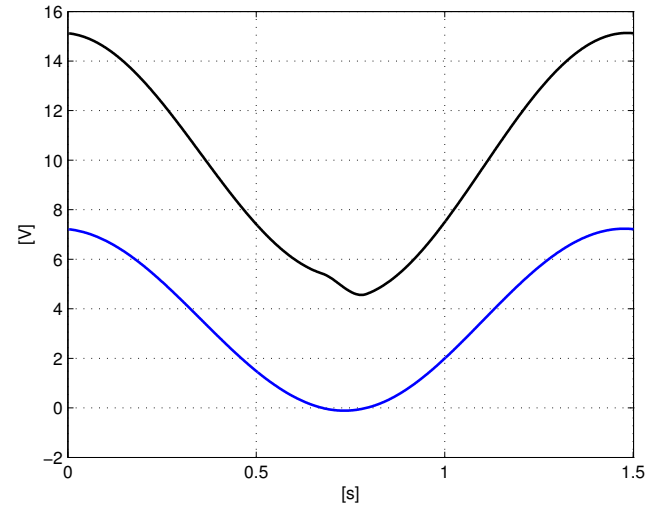

(a)

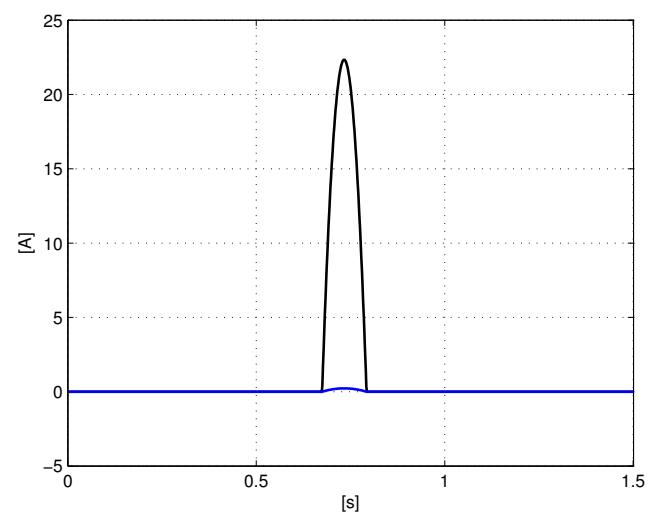

(b)

Fig. 5. Steady-state oscillations computed with $(\theta=0.5, \gamma=0.5)$ and by fixing $N_{\max }=900$ : (a) collector voltage (black line) and emitter voltage (blue line) (b) collector current $i_{C}$ (black line) and base current $i_{B}$ (blue line).

samples decreases when the number of samples increases. Future work will investigate the use of the proposed tool for the computation of limit cycles bifurcation diagrams.

\section{REFERENCES}

[1] T. Aprille and T. Trick, "A computer algorithm to determine the steadystate response of nonlinear oscillators," IEEE Trans. on Circuit Theory, vol. 19 , no. 4 , pp. 354-360, 1972.

[2] J. R. Parkhurst and L. L. Ogborn, "Determining the steady-state output of nonlinear oscillatory circuits using multiple shooting," IEEE Trans. Comput.-Aided Design Integr. Circuits Syst., vol. 14, no. 7, pp. 882888, 1995.

[3] D. Li and J. Xu, "A new method to determine the periodic orbit of a nonlinear dynamic systems and its period," Engineering with Computers, vol. 20, pp. 316-322, 2005.

[4] C. Theodosiou, A. Pournaras, and S. Natsiavas, "On periodic steady state response and stability of Filippov-type mechanical models," Nonlinear Dyn., vol. 66, pp. 355-376, 2011.

[5] R. I. Leine and H. Nijmeijer, Dynamics and Bifurcations of NonSmooth Mechanical Systems. London, UK: Springer Verlag,, 2004.

[6] F. Bonani and M. Gilli, "Analysis of stability and bifurcations of limit cycle in Chua's circuit through the Harmonic-Balance approach," IEEE Trans. Circuits Syst. I, Reg. Papers, vol. 46, no. 8, pp. 881-890, 1999.

[7] S. Engelberg, "Limitations of the describing function for limit cycle prediction," IEEE Trans. Autom. Control, vol. 47, no. 11, pp. 18871890, 2002.

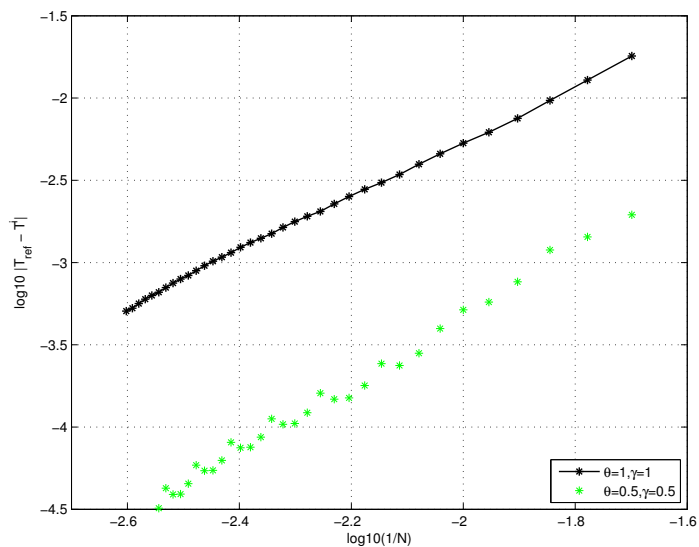

Fig. 6. Colpitts oscillator. Error in the period computation obtained with $N \in[50,300]$.

[8] M. Bonnin, M. Gilli, and P. P. Civalleri, "A mixed time-frequencydomain approach for the analysis of a hysteretic oscillator," IEEE Trans. Circuits Syst. II, Exp. Briefs, vol. 52, no. 9, pp. 525-529, 2005.

[9] F. Vasca, L. Iannelli, and M. K. Camlibel, "A new perspective for modeling power electronics converters: complementarity framework," IEEE Trans. Power Electronics, vol. 24, no. 2, pp. 456-468, 2009.

[10] J. M. Schumacher, "Complementarity systems in optimization," Mathematical Programming, vol. 101, no. 1, pp. 4263-296, 2004.

[11] L. Iannelli and F. Vasca, "Computation of limit cycles and forced oscillations in discrete-time piecewise linear feedback systems through a complementarity approach," in 47th IEEE Conference on Decision and Control, Cancun, Mexico, dec. 2008, pp. 1169-1174.

[12] L. Iannelli, F. Vasca, and V. Sessa, "Computation of limit cycles in Lur'e systems," in American Control Conference, San Francisco, CA, USA, june 2011, pp. 1402-1407.

[13] V. Sessa, L. Iannelli, and F. Vasca, "Mixed linear complementarity problems for the analysis of limit cycles in piecewise linear systems," in the 51st IEEE Conference on Decision and Control, Maui, Hawaii, USA, dec. 2012, pp. $1023-1028$.

[14] S. P. Dirkse and M. C. Ferris, "The PATH solver: a non-monotone stabilization scheme for mixed complementarity problems," Optimization Methods and Software, vol. 5, pp. 123-156, 1995.

[15] D. M. W. Leenaearts and W. van Bokhoven, Piecewise linear modeling and analysis. Kluwer Academic Publishers, Norwell, MA, USA, 1998.

[16] E. J. Doedel, "AUTO: A program for the automatic bifurcation analysis of autonomous systems," Congressus Numeratium, vol. 30, pp. 1265 $1284,1981$.

[17] A. H. Nayfeh and B. Balachandran, Applied Nonlinear Dynamics; Analytical, Computational and Experimental Methods. WileyInternational, Chichester, 1995.

[18] R. U. Seydel, Practical Bifurcation and Stability Analysis, third edition ed., ser. Interdisciplinary Applied Mathematics. New York: Springer, 1988, vol. 5.

[19] V. Acary, "Time-stepping via complementarity," in Dynamics and Control of Switched Electroinic Systems, ser. Advances in Industrial Control, F. Vasca and L. Iannelli, Eds. London, U.K.: Springer-Verlag, 2012.

[20] V. Acary and B. Brogliato, Numerical methods for nonsmooth dynamical systems. Berlin Heidelberg: Springer-Verlag, 2008, vol. 35.

[21] M. Di Bernardo, K. H. Johansson, and F. Vasca, "Self-oscillations and sliding in relay feedback systems: Symmetry and bifurcations," Int. J. Bifurcation \& Chaos, vol. 11, no. 4, pp. 1121-1140, 2001.

[22] A. Buonomo and A. Lo Schiavo, "Numerical analysis of the steadystate response of nonlinear circuits using the trigonometric collocation method," IEE Proceedings - Circuits, Devices and Systems, vol. 148, no. 1 , pp. 45-52, 2001 